\title{
Sparse Signal Reconstruction Based on Multiparameter Approximation Function with Smoothed $\ell_{0}$ Norm
}

\author{
Xiao-Feng Fang, ${ }^{1,2}$ Jiang-She Zhang, ${ }^{1}$ and Ying-Qi $\mathbf{L i}^{2}$ \\ ${ }^{1}$ School of Mathematics and Statistics, Xian Jiaotong University, Xian 710049, China \\ ${ }^{2}$ Mathematics and OR Section, Xi'an Research Institute of High-Tech, Hongqing Town, Xi'an 710025, China \\ Correspondence should be addressed to Jiang-She Zhang; jszhang@mail.xjtu.edu.cn
}

Received 26 December 2013; Revised 19 March 2014; Accepted 19 March 2014; Published 4 June 2014

Academic Editor: Xi-Ming Sun

Copyright (c) 2014 Xiao-Feng Fang et al. This is an open access article distributed under the Creative Commons Attribution License, which permits unrestricted use, distribution, and reproduction in any medium, provided the original work is properly cited.

The smoothed $\ell_{0}$ norm algorithm is a reconstruction algorithm in compressive sensing based on approximate smoothed $\ell_{0}$ norm. It introduces a sequence of smoothed functions to approximate the $\ell_{0}$ norm and approaches the solution using the specific iteration process with the steepest method. In order to choose an appropriate sequence of smoothed function and solve the optimization problem effectively, we employ approximate hyperbolic tangent multiparameter function as the approximation to the big "steep nature" in $\ell_{0}$ norm. Simultaneously, we propose an algorithm based on minimizing a reweighted approximate $\ell_{0}$ norm in the null space of the measurement matrix. The unconstrained optimization involved is performed by using a modified quasi-Newton algorithm. The numerical simulation results show that the proposed algorithms yield improved signal reconstruction quality and performance.

\section{Introduction}

Sparse representation of signals has been extensively studied for decades. The concept of signal sparsity and $\ell_{1}$ norm based on recovery techniques can be traced back to the work of Logan [1] in 1965, Santosa and Symes [2] in 1986, and Donoho and Stark in 1989 [3]. It is generally agreed that the foundation of the current state of the art in compressive sensing (CS) theory was laid by Candés et al. [4], Donoho [5], and Candés and Tao [6] in 2006. Mathematically, under sparsity assumptions, one would want to recover a signal $x \in$ $\mathbb{R}^{n}$, for example, the coefficient sequence of the signal in the appropriate basis, by solving the combinatorial optimization problem:

$$
\min _{x}\|x\|_{0} \quad \text { s.t. } y=\Psi x
$$

where $y$ is an observed signal, $\Psi$ is the dictionary matrix, $x$ means a coefficient vector for the linear combination, and $\|x\|_{0}$ is the $\ell_{0}$ norm of the vector $x$ indicating the number of its nonzero elements.
Exactly solving (1) is reported to be NP-hard [5, 7]; thus a relaxed approach [8-10] was proposed to address this challenge. By replacing nonconvex $\ell_{0}$ norm with convex $\ell_{1}$ norm, the method finds optimal solution using convex optimization $[8,11-13]$. It was also introduced $[6,14-17]$ that, for many problems, the minimizing of the $\ell_{1}$ norm is equivalent to that of the $\ell_{0}$ norm under certain conditions. However, experimental results in [13] posed a strong question about the equivalence of minimizing both norms in practical problems. The sparse signal reconstruction (SSR) algorithm based on the optimization of a smoothed approximate $\ell_{0}$ norm is studied in [11] where simulation results are compared with corresponding results obtained with several existing SSR algorithms with respect to reconstruction performance and computational complexity. These results favor the use of the approximate $\ell_{0}$ norm.

In this paper, we present a new signal reconstruction algorithm for CS, which is based on the minimization of a smoothed approximate $\ell_{0}$ norm. But it differs from the previous algorithms in several aspects. First, we use approximation hyperbolic tangent function to approximate 
the $\ell_{0}$ norm, which is found to have better approximation quality of $\ell_{0}$ norm than the standard Gauss function. Second, the $\ell_{0}$ norm minimization algorithm in this paper is carried out in null space of the measurement matrix, in which the constraints on measurements are eliminated and become unconstrained. Third, we use a reweighting technique in the null space of the measurement matrix to force the algorithm to reach the desired sparse solution faster. The rest of the paper is organized as follows. In Section 2, the smoothed $\ell_{0}$ norm algorithm is briefly presented. Section 3 is about the signal reconstruction on the base of the reweighted approximation smoothed $\ell_{0}$ norm algorithm. Section 4 provides the experimental results. The paper is finally concluded in Section 5.

\section{Smoothed $\ell_{0}$ Norm Algorithm (SLO)}

Smoothed $\ell_{0}$ norm attempts to solve the problem in (1) by approximating the $\ell_{0}$ norm with a continuous function. Consider the continuous Gaussian function $f_{\sigma}(x)$ with the parameter $\sigma$ :

$$
f_{\sigma}(x)=e^{-x^{2} / 2 \sigma^{2}}, \quad x \in \mathbb{R}, \sigma \in \mathbb{R}_{+} .
$$

The parameter $\sigma$ determines the quality of the approximation and for any family of functions $f_{\sigma}(x)$ which approximates the Kronecker delta function.

Define the continuous multivariate function $g(x)$ as

$$
g(x)=\sum_{i=1}^{N} f_{\sigma}\left(x_{i}\right), \quad x \in \mathbb{R}^{N \times 1}
$$

It follows from (2) and (3) that $\sigma \rightarrow 0$ and

$$
\lim _{\sigma \rightarrow 0} f_{\sigma}(x)= \begin{cases}1 & x=0 \\ 0 & x \neq 0\end{cases}
$$

Since the number of entries in $x$ is $N$ and the function $g(x)$ is an indicator of the number of zero entries in $x$, the $\ell_{0}$ norm of reconstructed vector $x$ is approximated by

$$
\|x\|_{0} \approx N-g(x) \text {. }
$$
lem

Substituting this approximation into (1) yields the prob-

$$
\begin{array}{ll}
\operatorname{minimise} & \sum_{i=1}^{N}\left(1-e^{-x_{i}^{2} / 2 \sigma^{2}}\right) \\
\text { s.t. } & y=\Psi x .
\end{array}
$$

The approach is then to solve the problem (6) for a decreasing sequence of $\sigma$ 's. The underlying thought is to select a $\sigma$ which ensures that the initial solution is in the subset of $\mathbb{R}^{N \times 1}$ over which the approximation is convex and gradually increases the accuracy of the approximation. Here, the final smoothed $\ell_{0}$ algorithm is proved to be faster with the possibility of recovering a sparser solution [11].
Algorithm 1. The final smoothed $\ell_{0}$ norm (SL0 algorithm) is as follows.

(1) Initialization: choose an arbitrary solution from the feasible set, for example, the minimum $\ell_{2}$ norm solution of $x=\Psi x$; that is,

$$
x=\Psi^{T}\left(\Psi \Psi^{T}\right)^{-1} y
$$

(2) Choose a decreasing sequence for $\sigma_{i}, i=1,2, \ldots, K$.

(3) For $k=1,2, \ldots, K$, let $\sigma=\sigma_{k}$. Minimize function (6) using $L$ iterations of the steepest ascent algorithm.

(a) Let $\Delta x=\left[x_{1} \exp \left(-x_{1}^{2} / 2 \sigma_{k}^{2}\right), \ldots, x_{n} \exp \left(-x_{n}^{2} /\right.\right.$ $\left.\left.2 \sigma_{k}^{2}\right)\right]^{T}$.

(b) Let $x \leftarrow x-\mu \Delta x$ (where $\mu$ is a small position constant); then

$$
x \longleftarrow x-\Psi^{T}\left(\Psi \Psi^{T}\right)^{-1} y .
$$

(4) Final answer is $x$.

\section{Signal Reconstruction Based on Approximation Function}

3.1. Approximate $\ell_{0}$ Norm with Approximate Hyperbolic Tangent Sequence. The key to SL0 algorithm is to select high quality smoothed continuous function to approximate $\ell_{0}$ norm. The optimal value of $\ell_{0}$ norm is achieved through the solution of the minimum value of the approximate smoothed continuous function. In general, the approximation of the $\ell_{0}$ norm with Gauss function cannot produce desired result. To effectively approximate $\ell_{0}$ norm, we analyzed inverse tangent function [18], hyperbolic tangent function, and approximate hyperbolic tangent sequence.

Inverse tangent function is as follows:

$$
h_{\sigma}\left(x_{i}\right)=\frac{2}{\pi} \arctan \left(\frac{x_{i}^{2}}{2 \sigma^{2}}\right) .
$$

Hyperbolic tangent function is as follows:

$$
g_{\sigma}\left(x_{i}\right)=\frac{e^{x_{i}^{2} / 2 \sigma^{2}}-e^{-x_{i}^{2} / 2 \sigma^{2}}}{e^{x_{i}^{2} / 2 \sigma^{2}}+e^{-x_{i}^{2} / 2 \sigma^{2}}} .
$$

Approximate hyperbolic tangent function is as follows:

$$
f_{\sigma}\left(x_{i}\right)=\frac{1}{8} \frac{e^{x_{i}^{2} / 2 \sigma^{2}}-e^{-x_{i}^{2} / 2 \sigma^{2}}}{e^{x_{i}^{2} / 2 \sigma^{2}}+e^{-x_{i}^{2} / 2 \sigma^{2}}}+\frac{7}{8}\left(1-e^{-x_{i}^{2} /\left((1 / 8) \sigma^{2}\right)}\right),
$$

where $\sigma$ is a parameter and $x_{i}$ is a component of the vector $x$. The following properties can be obtained. 
(1) For formula (11), let $F_{\sigma}(x)=\sum_{i=1}^{N} f_{\sigma}\left(x_{i}\right)$; then $\|x\|_{0}=$ $\lim _{\sigma \rightarrow 0} F_{\sigma}(x)$ since

$$
\begin{aligned}
& \lim _{\sigma \rightarrow 0} f_{\sigma}\left(x_{i}\right) \\
& =\lim _{\sigma \rightarrow 0}\left(\frac{1}{8} \cdot \frac{e^{x_{i}^{2} / 2 \sigma^{2}}-e^{-x_{i}^{2} / 2 \sigma^{2}}}{e^{x_{i}^{2} / 2 \sigma^{2}}+e^{-x_{i}^{2} / 2 \sigma^{2}}}+\frac{7}{8} \cdot\left(1-e^{-x_{i}^{2} /\left((1 / 8) \sigma^{2}\right)}\right)\right) \\
& = \begin{cases}0 & x_{i}=0 \\
1 & x_{i} \neq 0 .\end{cases}
\end{aligned}
$$

According to the definition of $\ell_{0}$ norm, when $\sigma$ goes infinitely to zero, the value of $F_{\sigma}(x)$ goes as well to the nonzero elements of the vector $x$, that is, the $\ell_{0}$ norm of vector $x$. Therefore, it can be shown with the smoothed function $\|x\|_{0}=\lim _{\sigma \rightarrow 0} F_{\sigma}(x)$. Obviously, formulas (9) and (10) have the same approximation property of $\ell_{0}$ norm as that of (11). The approximation performance of $\ell_{0}$ norm of each formula, however, is different. For further illustration of the advantages of approximate hyperbolic tangent function as smoothed continuous function, we compared the distribution of each approximation function at interval $[-1,1]$ when $\sigma=0.1$. Figure 1 is the approximation quality comparison of the smoothed $\ell_{0}$ norm of the standard Gauss function, inverse tangent function, hyperbolic tangent function, and approximate hyperbolic tangent function. The estimation of $\ell_{0}$ norm by approximate hyperbolic tangent function outsmarted other functions. It shows that the approximate hyperbolic tangent function has better "steep nature" between the intervals of $[-0.5,0.5]$ and the estimation of the $\ell_{0}$ norm is more accurate.

(2) Given $\sigma \geq 0$, approximate hyperbolic tangent function $f_{\sigma}\left(x_{i}\right)$ has better convergence than other approximate functions; that is, $f_{\sigma}\left(x_{i}\right) \geq\left(1-\varphi\left(x_{i}\right)\right)$, for arbitrary $\sigma, \varphi$, where $\varphi\left(x_{i}\right)=e^{-x_{i}^{2} / 2 \sigma^{2}}$.

Proof. Let $u(x)=f(x)-(1-\varphi(x))$, when $x=0$ or $x=\infty$, and $u(x)=0$, when $0<|x|<\infty$,

$$
\begin{aligned}
u(x)= & \frac{1}{8} \cdot \frac{e^{x^{2} / 2 \sigma^{2}}-e^{-x^{2} / 2 \sigma^{2}}}{e^{x^{2} / 2 \sigma^{2}}+e^{-x^{2} / 2 \sigma^{2}}} \\
& +\frac{7}{8} \cdot\left(1-e^{-x^{2} /\left((1 / 8) \sigma^{2}\right)}\right)-1+e^{-x^{2} / 2 \sigma^{2}} \\
= & \frac{1}{8} \cdot\left(1-\frac{2 e^{-x^{2} / 2 \sigma^{2}}}{e^{x^{2} / 2 \sigma^{2}}+e^{-x^{2} / 2 \sigma^{2}}}\right) \\
& +\frac{7}{8} \cdot\left(1-e^{-x^{2} /\left((1 / 8) \sigma^{2}\right)}\right)-1+e^{-x^{2} / 2 \sigma^{2}} \\
= & -\frac{1}{8} \cdot \frac{2 e^{-x^{2} / 2 \sigma^{2}}}{e^{x^{2} / 2 \sigma^{2}}+e^{-x^{2} / 2 \sigma^{2}}}-\frac{7}{8} \cdot e^{-x^{2} /\left((1 / 8) \sigma^{2}\right)} \\
& +\frac{1}{8} \cdot e^{-x^{2} / 2 \sigma^{2}}+\frac{7}{8} \cdot e^{-x^{2} / 2 \sigma^{2}}
\end{aligned}
$$

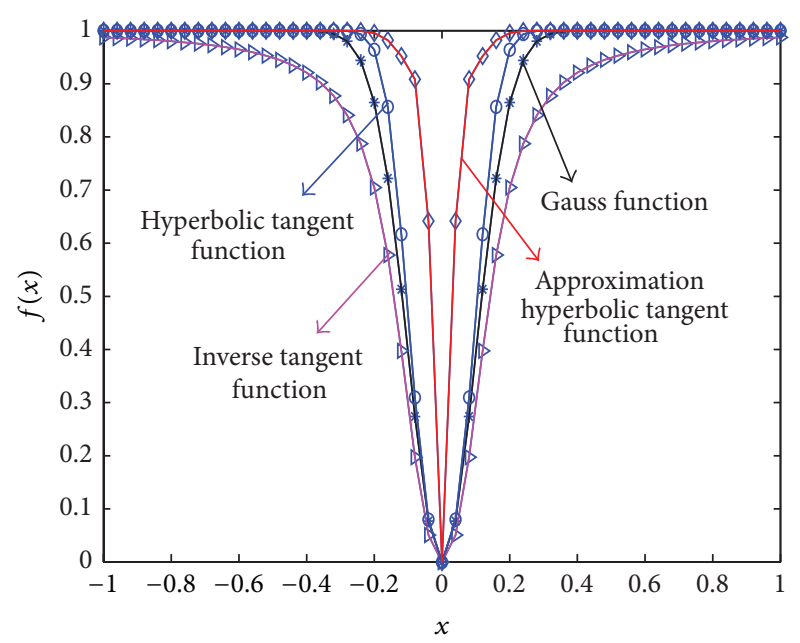

FIGURE 1: Comparison of the four approximate $\ell_{0}$ norm functions with $\sigma=0.1$.

$$
\begin{aligned}
& =\frac{1}{8} \cdot\left(\frac{-2 e^{-x^{2} / 2 \sigma^{2}}}{e^{x^{2} / 2 \sigma^{2}}+e^{-x^{2} / 2 \sigma^{2}}}+e^{-x^{2} / 2 \sigma^{2}}\right) \\
& +\frac{7}{8} \cdot\left(e^{-x^{2} / 2 \sigma^{2}}-e^{-x^{2} /\left((1 / 8) \sigma^{2}\right)}\right) \\
& =\frac{1}{8} \cdot \frac{e^{-x^{2} / 2 \sigma^{2}}}{e^{x^{2} / 2 \sigma^{2}}+e^{-x^{2} / 2 \sigma^{2}}} \cdot\left(-2+e^{x^{2} / 2 \sigma^{2}}+e^{-x^{2} / 2 \sigma^{2}}\right) \\
& +\frac{7}{8} \cdot\left(e^{-x^{2} / 2 \sigma^{2}}-e^{-x^{2} /\left((1 / 8) \sigma^{2}\right)}\right) \\
& \geq \frac{1}{8} \cdot \frac{1}{1+e^{x^{2} / \sigma^{2}}}\left(e^{x^{2} / 4 \sigma^{2}}+e^{-x^{2} / 4 \sigma^{2}}\right)^{2} \geq 0 .
\end{aligned}
$$

From (9), (10), and (11), it is clear that the smaller the $\sigma$ is, the more the local extreme values of objective function would be, and it would be difficult to get the global optimal value of the objective function. The $\sigma$ determines the smoothness of the objective function. The bigger the $\sigma$, the smoother the objective function, and the less accurate it would be to estimate the $\ell_{0}$ norm. Conversely, the smaller the $\sigma$ is, the better the approximation of the $\ell_{0}$ norm would be. In the process, we built a decreasing sequence $\sigma_{1}, \sigma_{2}, \ldots, \sigma_{n}$ to optimize each corresponding objective function until $\sigma_{i}$ is small enough, so as to eliminate the influence of the local extreme value and get the global optimal value of the smoothed function. We, thus, use approximate hyperbolic tangent sequence for the approximation of $\ell_{0}$ norm.

Problem (1) can be approximated by the following model:

$$
\min F_{\sigma}(x) \quad \text { s.t. } y=\Phi x .
$$

(3) There are lots of algorithms to the solving of problem (14), among which the most representative one is the steepest descent method. But there still exist two drawbacks. 
(a) "Notched effect" in search direction strongly hinders the convergence speed.

(b) Search steps cannot be estimated. Usually, it is estimated with experiences, which lacks theoretical support.

We, therefore, propose the modified quasi-Newton algorithm to solve the above problem. First, calculate the Newton direction of the approximate hyperbolic tangent function:

$$
d=-\nabla^{2} F_{\sigma}(x)^{-1} \nabla F_{\sigma}(x)
$$

where

$$
\begin{aligned}
& \nabla F_{\sigma}(x) \\
& =\left[\frac{1}{8} \cdot \frac{4 x_{1} / \sigma^{2}}{\left(e^{x_{1}^{2} / 2 \sigma^{2}}+e^{-x_{1}^{2} / 2 \sigma^{2}}\right)^{2}}\right. \\
& +\frac{7}{8} \cdot \frac{16 x_{1}}{\sigma^{2}} e^{-8 x_{1}^{2} / \sigma^{2}}, \ldots, \frac{1}{8} \cdot \frac{4 x_{N} / \sigma^{2}}{\left(e^{x_{N}^{2} / 2 \sigma^{2}}+e^{-x_{N}^{2} / 2 \sigma^{2}}\right)^{2}} \\
& \left.+\frac{7}{8} \cdot \frac{16 x_{N}}{\sigma^{2}} e^{-8 x_{N}^{2} / \sigma^{2}}\right]^{T} \\
& \nabla^{2}\left(F_{\sigma}(x)\right)=\left[\begin{array}{cccc}
\frac{\partial^{2} f_{\sigma}\left(x_{1}\right)}{\partial x_{1}^{2}} & 0 & \cdots & 0 \\
0 & \frac{\partial^{2} f_{\sigma}\left(x_{2}\right)}{\partial x_{2}^{2}} & \cdots & 0 \\
\vdots & \vdots & \vdots & \vdots \\
0 & 0 & \cdots & \frac{\partial^{2} f_{\sigma}\left(x_{N}\right)}{\partial x_{N}^{2}}
\end{array}\right] \text {, } \\
& \frac{\partial^{2} f_{\sigma}\left(x_{k}\right)}{\partial x_{k}^{2}}=\frac{1}{8} \cdot \frac{\left(4 / \sigma^{2}\right)\left(1+\left(x_{k}^{2} / \sigma^{2}\right)\right)}{\left(e^{x_{k}^{2} / 2 \sigma^{2}}+e^{-x_{k}^{2} / 2 \sigma^{2}}\right)^{2}} \\
& +\frac{7}{8} \cdot \frac{16}{\sigma^{2}} \sigma^{-8 x_{k}^{2} / \sigma^{2}}\left(1-\frac{16 x_{k}^{2}}{\sigma^{2}}\right) \text {. }
\end{aligned}
$$

We know that the Hesse matrix is nonpositive definite in Newton direction $d$, which cannot ensure that the Newton direction is descent direction, so the Hesse matrix should be modified. Then, set up a new matrix:

$$
G=\nabla^{2}\left(F_{\sigma}(x)\right)+\varepsilon_{k} I,
$$

where $\varepsilon_{k}$ is a group of proper positive numbers, $I$ is identity matrix, and the diagonal elements in matrix $G$ are all positive. Since

$$
\lim _{x_{k} \rightarrow 0}\left(e^{x_{k}^{2} / 2 \sigma^{2}}+e^{-x_{k}^{2} / 2 \sigma^{2}}\right)^{2}=4
$$

choose

$$
\varepsilon_{k}=\frac{7}{8} \cdot \frac{16^{2} x_{k}^{2}}{\sigma^{4}} e^{-8 x_{k}^{2} / \sigma^{2}},
$$

as modification coefficients; then the diagonal elements in matrix $G$ can be shown as

$$
\frac{1}{8} \cdot \frac{4\left(1+\left(x_{k}^{2} / \sigma^{2}\right)\right)}{\sigma^{2}\left(e^{x_{k}^{2} / 2 \sigma^{2}}+e^{-x_{k}^{2} / 2 \sigma^{2}}\right)^{2}}+\frac{7}{8} \cdot \frac{16}{\sigma^{2}} e^{-8 x_{k}^{2} / \sigma^{2}} .
$$

Also, it can be obtained that

$$
\begin{aligned}
d= & -\nabla^{2} F_{\sigma}(x)^{-1} \cdot \nabla F_{\sigma}(x) \\
= & {\left[-\frac{\sigma^{2} x_{1}+28 \cdot 4 \sigma^{2} x_{1} e^{-8 x_{1}^{2} / \sigma^{2}}}{\sigma^{2}+x_{1}^{2}+28 \cdot 4 \sigma^{2} e^{-8 x_{1}^{2} / \sigma^{2}}}, \ldots,\right.} \\
& \left.\quad-\frac{\sigma^{2} x_{N}+28 \cdot 4 \sigma^{2} x_{N} e^{-8 x_{N}^{2} / \sigma^{2}}}{\sigma^{2}+x_{N}^{2}+28 \cdot 4 \sigma^{2} e^{-8 x_{N}^{2} / \sigma^{2}}}\right]^{T} .
\end{aligned}
$$

(4) Usually, parameter $\sigma$ is chosen as $\sigma_{j}=\gamma \cdot \sigma_{j-1}, j=$ $1,2, \ldots$, and $J, \gamma \in(0.5,1) . \sigma_{1}$ and $\sigma_{j}$ are chosen as follows.

Let $x=\max _{i}\left|x_{i}^{0}\right|$, for faster algorithm convergence; parameter $\sigma$ should meet the following:

$$
h_{\sigma_{1}}(x)=\frac{2}{\pi} \arctan \left(\frac{x^{2}}{2 \sigma_{1}^{2}}\right) \leq \frac{1}{2} \Longrightarrow \sigma_{1} \geq \frac{x}{\sqrt{2}},
$$

and choosing $\sigma_{1}=\max _{i}\left(\left|x_{i}^{0}\right| / \sqrt{2}\right)$, when $\sigma_{j} \rightarrow 0$, we can obtain $F_{\sigma_{j}}(x) \rightarrow\|x\|_{0}$, so when $\sigma_{j}$ is smaller, $F_{\sigma_{j}}(x)$ can better reflect the sparsity of vector $x$. Meanwhile, however, it is more sensitive to noise; the value of $\sigma_{j}$ should not be too small. In summary, the steps are as follows.

Algorithm 2. The approximate smoothed $\ell_{0}$ norm (ASL0 algorithm) is as follows.

(1) Initialization: $x=\Phi^{T} y$, initial residual $r_{0}=0$, and smoothed function's initial parameter $\sigma=1$.

(2) Calculate the modified Newton direction with (23) and use modified Newton algorithm to get $x=x+d$.

(3) Use gradient projection to have $x=x-$ $\Phi^{T}\left(\Phi \Phi^{T}\right)^{-1}(\Phi x-y)$, and calculate the residual $r=$ $y-\Phi x$.

(4) If the residual of the adjacent iterations meets $\| r-$ $r_{0} \| \leq \varepsilon$, then stop. Otherwise, let $r_{0}=r$, continue, and return to (2).

(5) Further decrease $\sigma$, let $\sigma=\gamma \cdot \sigma(0<\gamma<1)$, usually set $\gamma=0.7$, and return to (2). Otherwise, stop iterating and get the optimal value $x$.

3.2. Reweighting the Approximate $\ell_{0}$ Norm Minimization. It is well known that all solutions of $\Psi x=y$ can be parameterized as

$$
x=x_{s}+V_{n} \xi
$$


where $x_{s}$ is a solution of $\Psi x=y$ and $V_{n}$ is a matrix whose columns constitute an orthogonal basis of the null space of $\Psi$ [19]. Vector $x_{s}$ and matrix $V_{n}$ can be evaluated by using the singular-value decomposition (SVD) or by using the QR decomposition of matrix $\Psi$ [20]. Using (25) the problem in (14) is reduced to

$$
\underset{\xi}{\operatorname{minimize}} \sum_{i=1}^{N} f_{\sigma}\left(x_{s i}+V_{i}^{T} \xi\right),
$$

where

$$
\begin{aligned}
f_{\sigma}( & \left.x_{s i}+V_{i}^{T} \xi\right) \\
= & \frac{1}{8} \cdot \frac{e^{\left[x_{s i}+v_{i}^{T} \xi\right]^{2} / 2 \sigma^{2}}-e^{-\left[x_{s i}+v_{i}^{T} \xi\right]^{2} / 2 \sigma^{2}}}{e^{\left[x_{s i}+v_{i}^{T} \xi\right]^{2} / 2 \sigma^{2}}+e^{-\left[x_{s i}+v_{i}^{T} \xi\right]^{2} / 2 \sigma^{2}}} \\
& +\frac{7}{8} \cdot\left(1-e^{-\left[x_{s i}+v_{i}^{T} \xi\right]^{2} /\left((1 / 8) \sigma^{2}\right)}\right) .
\end{aligned}
$$

Signal reconstruction based on the solution of the problem in (28) works well, but the technique can be considerably enhanced by incorporating a reweighting strategy, and the reweighted unconstrained problem can be formulated as follows [21]:

$$
\underset{\xi}{\operatorname{minimize}} \sum_{i=1}^{N} w_{i} \cdot f_{\sigma}\left(x_{s i}+V_{i}^{T} \xi\right),
$$

where $w_{i}$ are positive scalars that are from a weight vector $w=\left[w_{1} w_{2} \cdots w_{N}\right]$. Starting with an initial $w^{0}=e_{N}$, where $e_{N}$ is the all-one vector of dimension $N$, in the $(k+1)$ th iteration the weight vector is updated to $w^{(k+1)}$ with its $i$ th component given by

$$
w_{i}^{(k+1)}=\frac{1}{\left|x_{i}^{(k)}\right|+\tau}
$$

where $x_{i}^{(k)}$ denotes the $i$ th component of vector $x^{(k)}$ obtained in the $k$ th iteration as $x^{(k)}=x_{s}+V_{n} \xi^{(k)}$ and $\tau$ is a small positive scalar which is used to prevent numerical instability when $\left|x_{i}^{(k)}\right|$ approaches zero. As long as $\sigma>0$, the objective function in (28) remains differentiable and its gradient can be obtained as

$$
\begin{aligned}
g_{i}(x)=w_{i}\left(\frac{1}{8}\right. & \frac{4\left[x_{s i}+v_{i}^{T} \xi\right]}{\sigma^{2}} \\
& \left.+\frac{7}{8} \cdot \frac{16\left[x_{s i}+v_{i}^{T} \xi\right]}{\sigma^{2}} e^{-8\left[x_{s i}+v_{i}^{T} \xi\right]^{2} / \sigma^{2}}\right) .
\end{aligned}
$$

For a fixed value of $\sigma$, the problem in (26) is solved by using a quasi-Newton algorithm where an approximation of the inverse of the Hessian is obtained by using the BroydenFletcher-Goldfarb-Shanno (BFGS) update formula. It can be shown that function (28) remains convex in the region where the largest magnitude of the components of $x=x_{l_{2}}+V_{n} \xi$ is less than $\sigma$, where vector $x_{s}$ is chosen to be the least squares solution $x_{l_{2}}$ of $y=\Psi x$; namely, $x_{s}=x_{l_{2}}=\Psi^{T}\left(\Psi \Psi^{T}\right)^{-1} y$. Based on this, a reasonable initial value of $\sigma$ can be chosen as $\sigma_{0}=\max \left|x_{l_{2}}\right|+\tau$, where $\tau$ is a small positive scalar. As the algorithm starts at the origin $\xi^{(0)}=0$, the above choice of $\sigma_{0}$ ensures that the optimization starts in a convex region. This greatly facilitates the convergence of the proposed algorithm.

Algorithm 3. The reweighted approximate smoothed $\ell_{0}$ norm (RASL0 algorithm) is as follows.

(1) Initialization: input $\Psi, x_{l_{2}}, \sigma_{T}, r, \tau$, and $\varepsilon$, and set $\xi^{(0)}=$ $0, w^{(0)}=e_{N}, \sigma=\max \left|x_{l_{2}}\right|+\tau$, and $k=0$.

(2) Perform the $Q R$ decomposition of $\Psi^{T}=Q R$ and construct $V_{n}$ using the last columns of $Q$.

(3) With $w=w^{(k)}$ and $\xi^{(0)}$ as an initial point, apply the BFGS algorithm to solve the problem in (28), where reweighting with parameter $\varepsilon$ is applied using (29) in each iteration; denote the solution by $\xi^{(k)}$.

(4) Compute $x^{(k)}=x_{l_{2}}+V_{n} \xi^{(k)}$ and update weight vector to $w^{(k+1)}$ with (29).

(5) If $\sigma \leq \sigma_{T}$, stop and output $x^{(k)}$ as the solution; otherwise set $\xi^{(0)}=\xi^{(k)}, \sigma=r \cdot \sigma, k=k+1$, and repeat from step (3).

\section{Simulation Result}

This section describes some experiments testifying to the performances of the proposed algorithm. All experiments are performed under Windows 7 and MATLAB V8.0 (R2012a) running on a Lenovo workstation with an Intel (R) Core (TM), CPU i7 at $2.40 \mathrm{GHz}$, and $6 \mathrm{~GB}$ of memory.

Simulation 1: Algorithm Performances Comparison of OneDimensional Signal. In the experiment, the signal length and number of measurements were set to $N=256$ and $M=100$, respectively. A total of 16 sparse signals with sparsity $K=$ $4 q-3, q=1,2, \ldots, 16$, were used. Then a $K$-sparse signal $x$ was constructed as follows.

(1) Set $x$ to zero vector of length $N$.

(2) Generate a vector $u$ of length $K$ assuming that each component $u_{i}$ is a random value drawn from a normal distribution $N(0,1)$.

(3) Randomly select $K$ indices from the set $1,2, \ldots, N$, and set $x_{i 1}=u_{1}$, and $x_{i 2}=u_{2}, \ldots, x_{i K}=u_{K}$. The measurement matrix was assumed to be of size $M \times$ $N$ and was generated by drawing its elements from $N(0,1)$, followed by a normalization step to ensure that the $\ell_{2}$ norm of each column is unity.

The performance of iteratively reweighted (IR) algorithm $[22,23]$ with $p=0.1$, the SL0 algorithm, ASL0 algorithm and RASL0 algorithm with $\sigma_{T}=10^{-4}, r=1 / 3, \tau=0.01$, and $\epsilon=0.08$ was measured in terms of the number of perfect reconstructions over 100 runs. The results obtained were plotted in Figure 2. It can be observed that the RASL0 


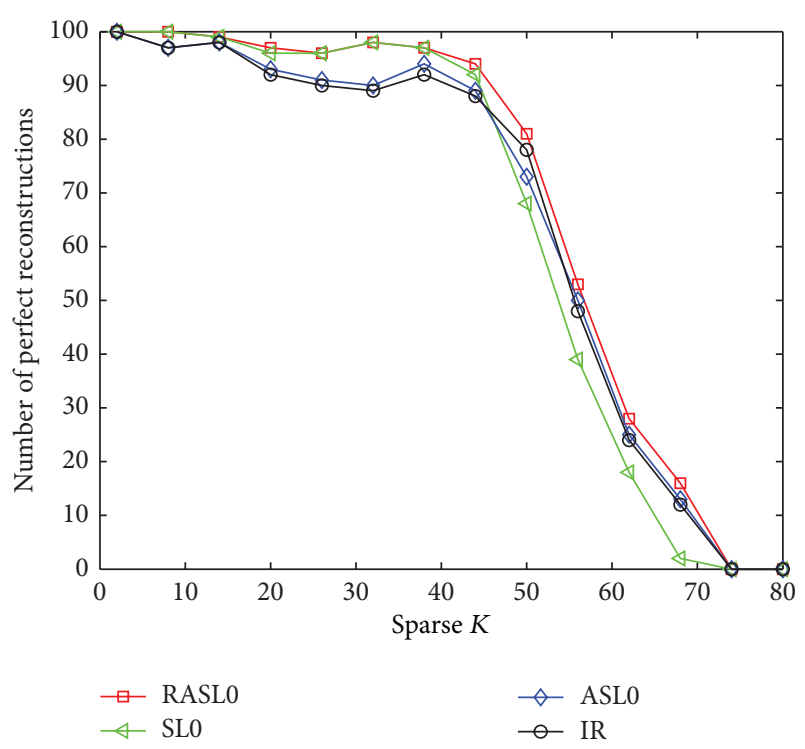

FIGURE 2: Number of perfect reconstructions by each algorithm over 100 runs with $N=256$ and $M=100$.

algorithm outperforms the IR algorithm. On comparing the SL0 algorithm and the ASL0 algorithm, we note that the two algorithms are comparable for $K$ smaller than 45 but the RASL0 algorithm performs better for $K$ larger than 45.

The mathematical complexity of the SL0 algorithm, IR algorithm, ASL0 algorithm, and RASL0 algorithm was measured in terms of average CPU time over 100 runs for typical instances with $M=N / 2$ and $K=\operatorname{round}(M / 2.5)$ where $N$ varies in the range between 128 and 512. In Figure 3, it is noted that the RASL0 algorithm and the SL0 algorithms were more efficient than the IR algorithm, and the complexity of the RASL0 algorithm was slightly higher than that of the SL0 algorithm. The moderate increase in the mathematical complexity of the RASL0 algorithm was primarily due to the fact that the objective function in (26) needed to be modified in each iteration using (29). Typically the RASL0 algorithm converges in a small number of iterations. Figure 4 showed how the objective function in (26) converges in 24 iterations, where the parameters were set to $N=256, M=100, K=40$, and $\sigma=0.0212$.

Simulation 2: Algorithm Performances Comparison of TwoDimensional Signal. As to examine the algorithms' performances for different images, the reconstruction performance of each method is evaluated in terms of two indicators: the reconstruction relative error (RE) and peak signal-to-noise ratio (PSNR). The relative error of reconstructed image is defined as follows:

$$
\mathrm{RE}(x)=\frac{\|x-\bar{x}\|_{2}}{\|\bar{x}\|_{2}}
$$

where $\bar{x}$ and $x$ are the original and the reconstructed image, respectively. Apparently, the lower the value of relative error

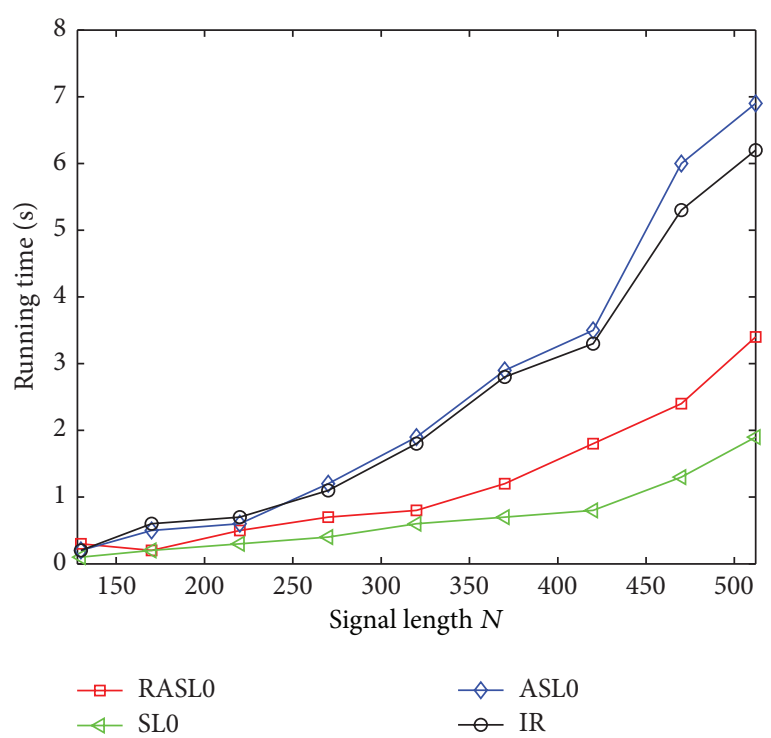

FIGURE 3: Average CPU time required by each algorithm over 100 runs with $M=N / 2$ and $K=\operatorname{round}(M / 2.5)$.

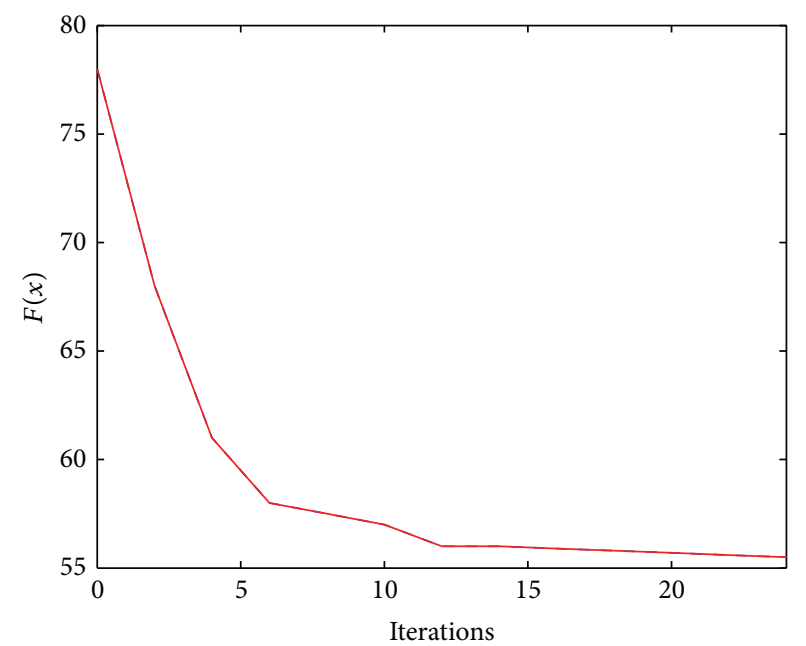

FIgURE 4: Function $F_{\sigma}(\xi)$ for $N=256, M=100, K=40$, and $\sigma=0.0212$.

is, the better the reconstructed performance will be. The PSNR of reconstructed images is defined by

$$
\operatorname{PSNR}(x)=10 \log _{10}\left(\frac{m \times n \times 255^{2}}{\|x-\bar{x}\|_{2}}\right),
$$

where $m, n$ are the size of the image.

In experiment, the DCT basis is selected as the sparse representation dictionary; compression ratio is $M / N=0.25$. Figure 5 shows that the quality of the reconstruction with RASL0 algorithm has been improved and details of images are better reconstructed.

Table 1 shows three different $256 \times 256$ images, with the same compression ratio $M / N=0.5$. From the comparison of the PSNR, the RE, the signal-to-noise ratio (SNR), and the matching degree (MD) among RASLO algorithm, SL0 


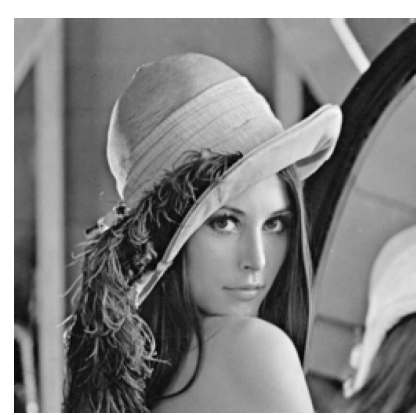

(a) Original image

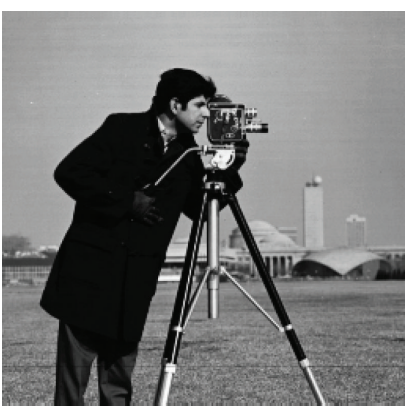

(e) Original image

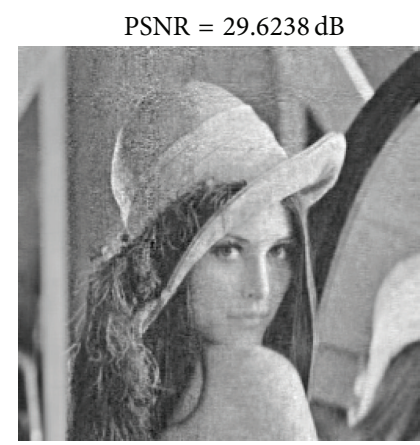

(b) SL0

$\mathrm{PSNR}=26.4476 \mathrm{~dB}$

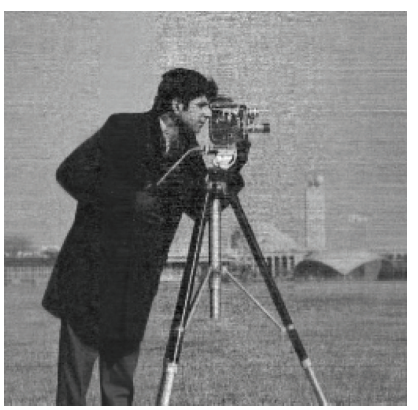

(f) SL0

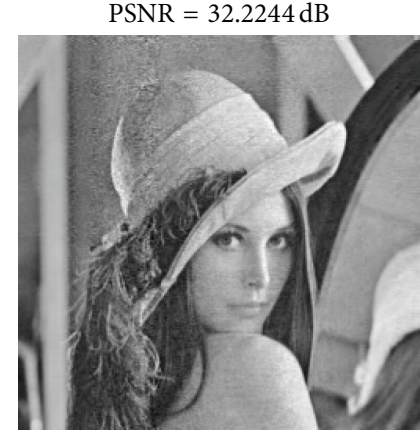

(c) ASL0

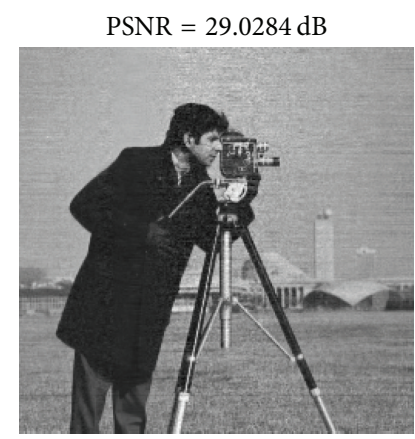

(g) ASL0

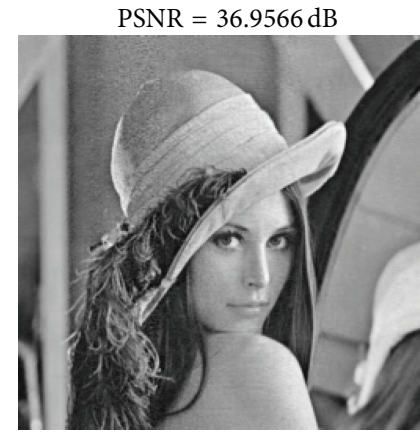

(d) RASLO

$P S N R=33.3116 \mathrm{~dB}$

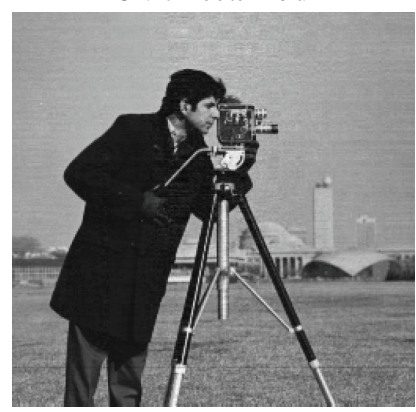

(h) RASL0

FIGURE 5: The quality of the reconstruction with different algorithms in different images. Original image.

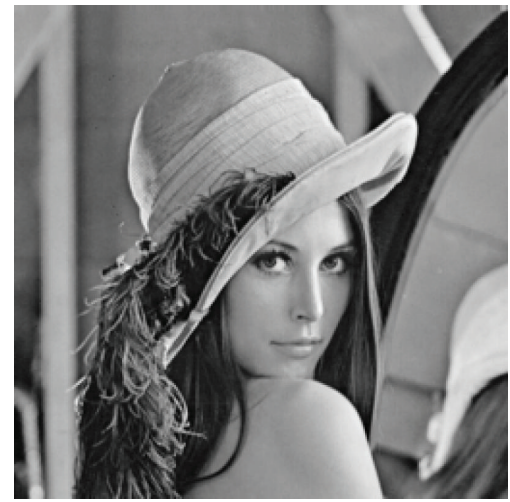

(a) Original image

PSNR $=31.2486 \mathrm{~dB}$

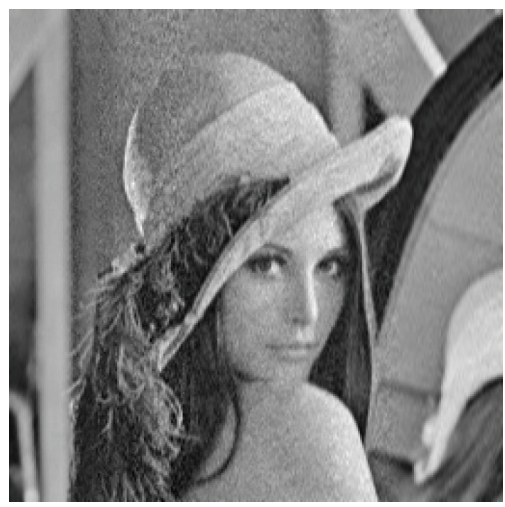

(d) SP

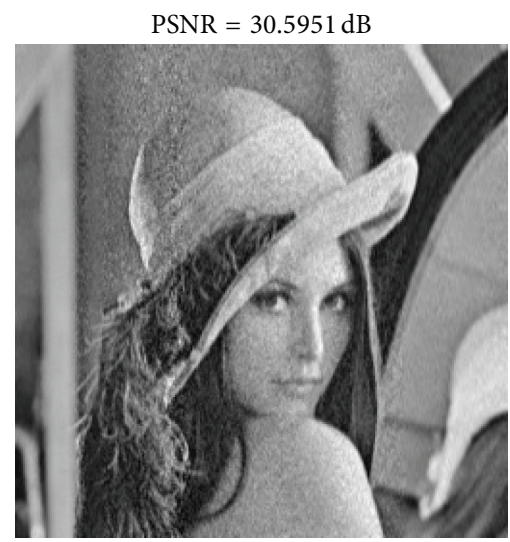

(b) OMP

PSNR $=34.2154 \mathrm{~dB}$

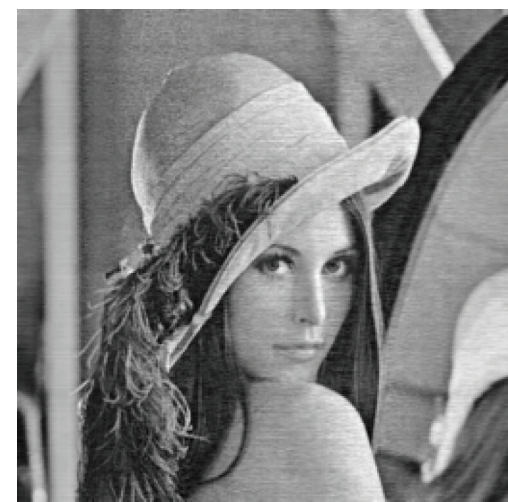

(e) GPSR

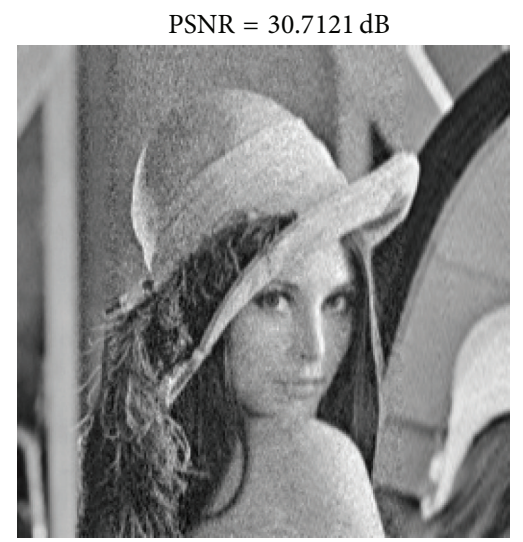

(c) GP

PSNR $=36.9566 \mathrm{~dB}$

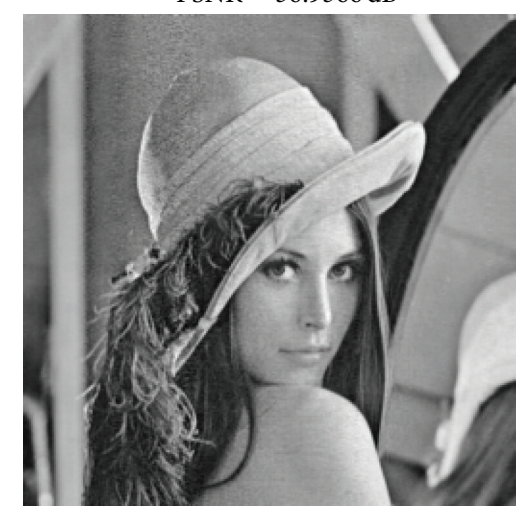

(f) RASL

FIGURE 6: Reconstruction quality comparison with four other algorithms. 
TABLE 1: Reconstruction quality comparison with different images and different approximation smoothed $\ell_{0}$ algorithms.

\begin{tabular}{|c|c|c|c|c|c|}
\hline Image & Algorithm & PSNR (dB) & $\mathrm{RE}$ & $\mathrm{SNR}(\mathrm{dB})$ & $\mathrm{MD}$ \\
\hline \multirow{3}{*}{ Lena } & SL0 & 29.6238 & 0.0947 & 34.3124 & 0.9831 \\
\hline & ASL0 & 32.2244 & 0.0742 & 36.2702 & 0.9893 \\
\hline & RASL0 & 36.9566 & 0.0578 & 39.9432 & 0.9926 \\
\hline \multirow{3}{*}{ Camera } & SL0 & 26.4476 & 0.0921 & 30.6337 & 0.9846 \\
\hline & ASL0 & 29.0284 & 0.0747 & 32.2134 & 0.9873 \\
\hline & RASL0 & 33.3116 & 0.0634 & 36.2727 & 0.9912 \\
\hline \multirow{3}{*}{ Boat } & SL0 & 28.1573 & 0.0852 & 31.3023 & 0.9813 \\
\hline & ASL0 & 29.5001 & 0.0786 & 33.7382 & 0.9865 \\
\hline & RASL0 & 30.5814 & 0.0614 & 34.3743 & 0.9908 \\
\hline
\end{tabular}

TABLE 2: Reconstruction quality comparison with four other algorithms.

\begin{tabular}{lccc}
\hline Algorithm & PSNR $(\mathrm{dB})$ & RE & Runtime $(\mathrm{s})$ \\
\hline OMP & 30.5951 & 0.0812 & 48.32 \\
GP & 30.7121 & 0.0803 & 12.23 \\
SP & 31.2486 & 0.0702 & 8.82 \\
GPSR & 34.2154 & 0.0656 & 11.38 \\
RASL0 & 36.9566 & 0.0578 & 13.36 \\
\hline
\end{tabular}

algorithm, and ASL0 algorithm, it is clear that the relative errors with RASL0 algorithm are $0.02 \%$ smaller than the SL0 algorithm, the SNR ratio is $3 \mathrm{~dB}$ higher, the PSNR is improved by $2 \mathrm{db}$, and the matching degree is also improved.

Figure 6 is the reconstruction effect comparison of image (Lena) with different algorithms. It shows that RASL0 outperforms OMP, GP, GPSR, and NSL0 algorithms. Table 2 is the quality comparison of different algorithms, indicating that RASL0 algorithm is better in all aspects.

\section{Conclusion and Future Work}

In this paper, we use approximate hyperbolic tangent function to estimate $\ell_{0}$ norm and design a RSNL0 algorithm based on minimizing an approximate $\ell_{0}$ norm of the signal in the null space of the measurement matrix, where a reweighting technique is used to force the solution's sparsity and a quasiNewton algorithm is used to accelerate the optimization. Simulation results are presented which demonstrate that the proposed algorithm yields improved signal reconstruction performance and requires a reduced amount of computation relative to iteratively reweighted algorithms based on the $\ell_{p}$ norm, with $p<1$. When compared with a known algorithm based on a smoothed $\ell_{0}$ norm, improved signal reconstruction is achieved although the amount of computation is increased somewhat. However, how to choose the parameter decreasing sequence so as to eliminate the disturbance of the minimal value to the solution of the minimum value would be our future work.

\section{Conflict of Interests}

The authors declare that there is no conflict of interests regarding the publication of this paper.

\section{Acknowledgments}

This work was supported by the National Basic Research Program of China (973 Program) under Grant no. 2013CB329404, the Major Research Project of the National Natural Science Foundation of China under Grant no. 91230101, the National Natural Science Foundation of China under Grant no. 61075006, and the Key Project of the National Natural Science Foundation of China under Grant no. 11131006 .

\section{References}

[1] B. F. Logan, Properties of high-pass signals [Ph.D. thesis], Columbia University, 1965.

[2] F. Santosa and W. W. Symes, "Linear inversion of band-limited reflection seismograms," Society for Industrial and Applied Mathematics, vol. 7, no. 4, pp. 1307-1330, 1986.

[3] D. L. Donoho and P. B. Stark, "Uncertainty principles and signal recovery," SIAM Journal on Applied Mathematics, vol. 49, no. 3, pp. 906-931, 1989.

[4] E. J. Candès, J. Romberg, and T. Tao, "Robust uncertainty principles: exact signal reconstruction from highly incomplete frequency information," IEEE Transactions on Information Theory, vol. 52, no. 2, pp. 489-509, 2006.

[5] D. L. Donoho, "Compressed sensing," IEEE Transactions on Information Theory, vol. 52, no. 4, pp. 1289-1306, 2006.

[6] E. J. Candès and T. Tao, "Near-optimal signal recovery from random projections: universal encoding strategies?" IEEE Transactions on Information Theory, vol. 52, no. 12, pp. 5406-5425, 2006.

[7] S. G. Mallat and Z. Zhang, "Matching pursuits with timefrequency dictionaries," IEEE Transactions on Signal Processing, vol. 41, no. 12, pp. 3397-3415, 1993.

[8] E. J. Candès, J. K. Romberg, and T. Tao, "Stable signal recovery from incomplete and inaccurate measurements," Communications on Pure and Applied Mathematics, vol. 59, no. 8, pp. 12071223, 2006.

[9] E. J. Candès and T. Tao, "The Dantzig selector: statistical estimation when $p$ is much larger than n," The Annals of Statistics, vol. 35, no. 6, pp. 2313-2351, 2007. 
[10] D. L. Donoho, "For most large undetermined systems of linear equations the minimal $l_{1}$-norm solutionis also the sparsest solution," Tech. Rep., 2004, http://www.stat.stanford.edu/donoho/ Reports.

[11] H. Mohimani, M. Babaie-Zadeh, and C. Jutten, "A fast approach for overcomplete sparse decomposition based on smoothed $\ell_{0}$ norm," IEEE Transactions on Signal Processing, vol. 57, no. 1, pp. 289-301, 2009.

[12] S. Boyd and L. Vandenberghe, Convex Optimization, Cambridge University Press, 2004.

[13] L. Mancera and J. Portilla, "L0-norm-based sparse representation through alternate projections," in Proceedings of the IEEE International Conference on Image Processing (ICIP '06), pp. 2089-2092, October 2006.

[14] H. Huang and A. Makur, "Backtracking-based matching pursuit method for sparse signal reconstruction," IEEE Signal Processing Letters, vol. 18, no. 7, pp. 391-394, 2011.

[15] T. T. Cai and A. Zhang, "Sharp RIP bound for sparse signal and low-rank matrix recovery," Applied and Computational Harmonic Analysis, vol. 35, no. 1, pp. 74-93, 2013.

[16] Y. C. Eldar and G. Kutyniok, Compressed Sensing, Cambridge University Press, 2012.

[17] E. Liu and V. N. Temlyakov, "The orthogonal super greedy algorithm and applications in compressed sensing," IEEE Transactions on Information Theory, vol. 58, no. 4, pp. 2040-2047, 2012.

[18] W. Jun-hua, H. Zhi-tao, Z. Yi-yu, and W. Feng-hua, "Robust sparse recovery based on approximate $l_{0}$ norm," Acta Electronica Sinica, vol. 40, no. 6, pp. 1185-1189, 2012.

[19] A. Antoniou and W.-S. Lu, Practical Optimization: Algorithms and Engineering Applications, Springer, 2007.

[20] G. H. Golub and C. F. van Loan, Matrix Computations, The Johns Hopkins University Press, 3rd edition, 1996.

[21] E. J. Candès, M. B. Wakin, and S. P. Boyd, "Enhancing sparsity by reweighted $l_{1}$ minimization," The Journal of Fourier Analysis and Applications, vol. 14, no. 5-6, pp. 877-905, 2008.

[22] P. C. Hansen and M. Saxild-Hansen, "A MATLAB package of algebraic iterative reconstruction methods," Journal of Computational and Applied Mathematics, vol. 236, no. 8, pp. 2167-2178, 2012.

[23] R. Chartrand and W. Yin, "Iteratively reweighted algorithms for compressive sensing," in Proceedings of the IEEE International Conference on Acoustics, Speech and Signal Processing (ICASSP '08), pp. 3869-3872, April 2008. 


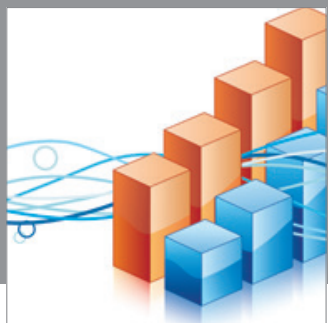

Advances in

Operations Research

mansans

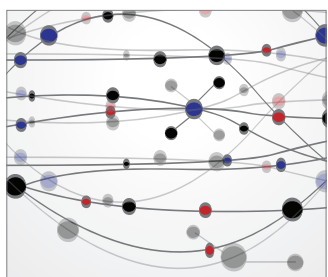

The Scientific World Journal
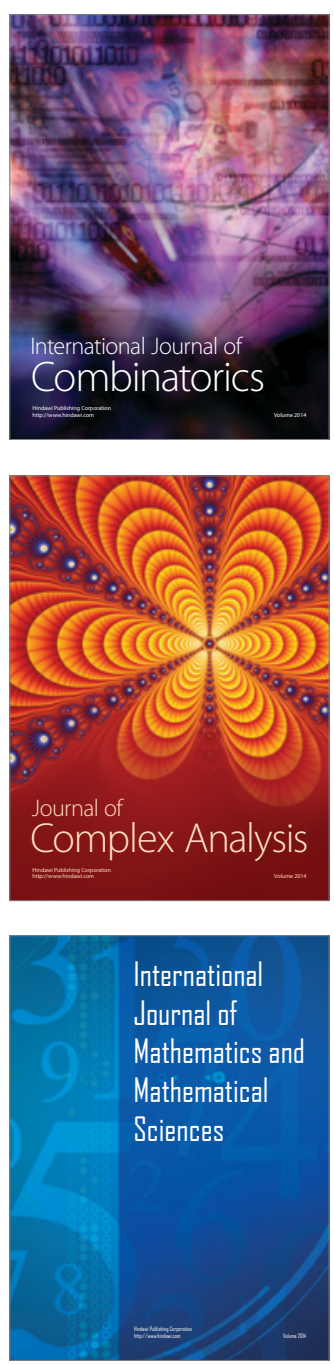
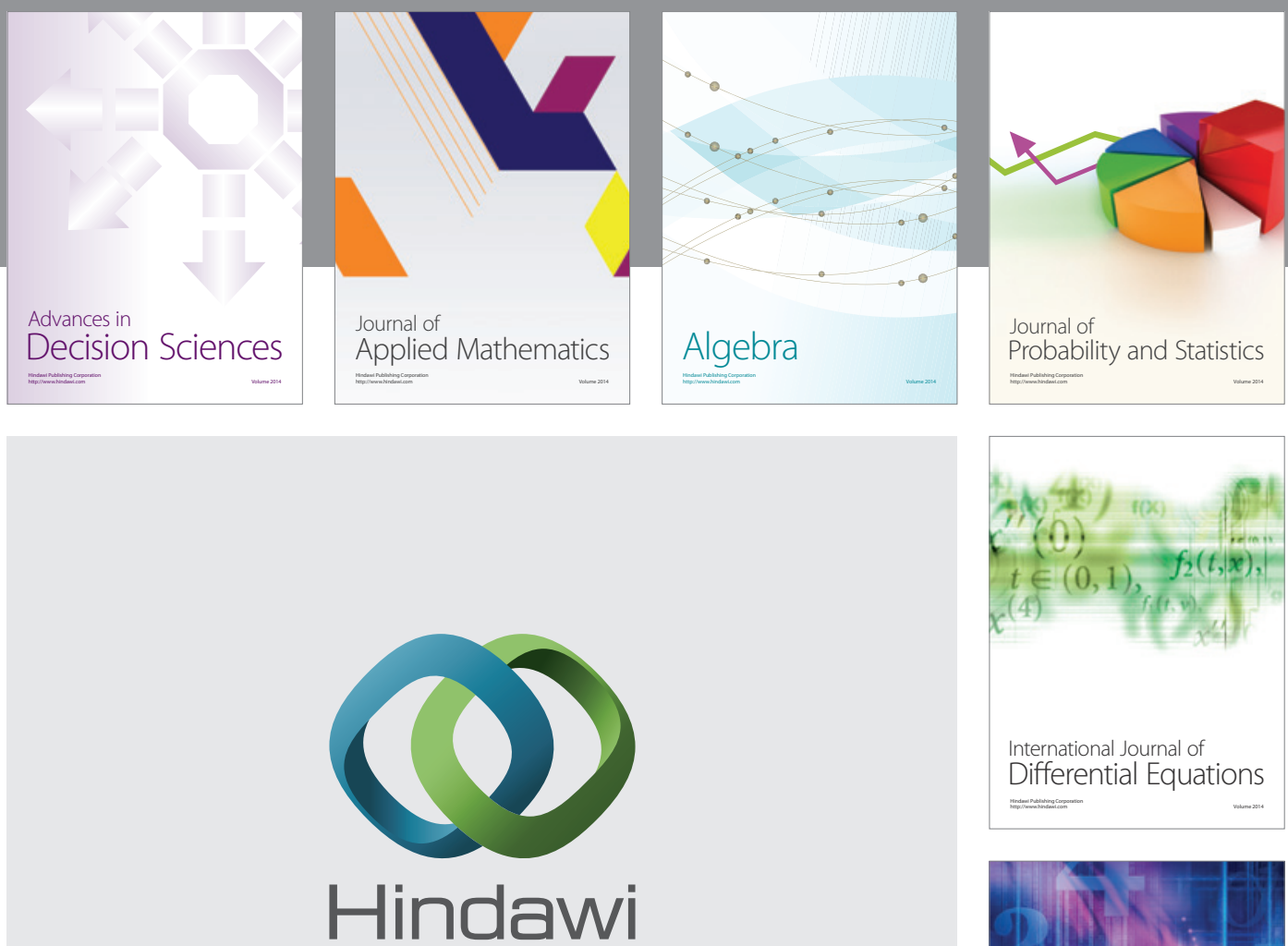

Submit your manuscripts at http://www.hindawi.com
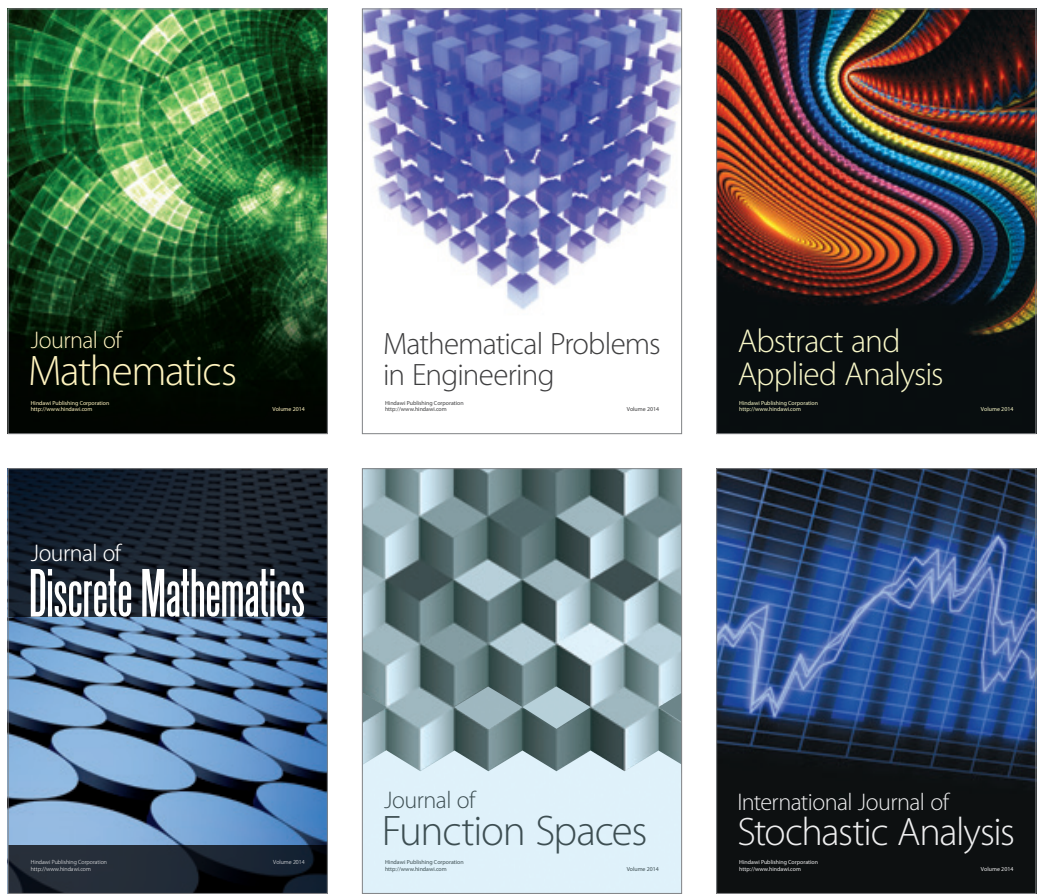

Journal of

Function Spaces

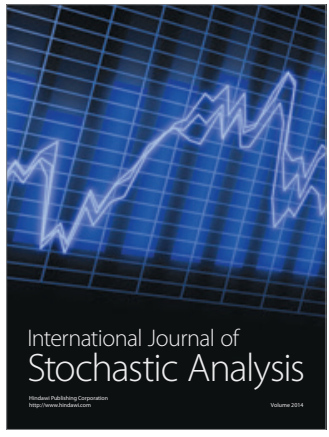

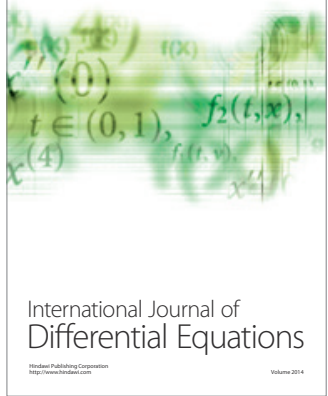
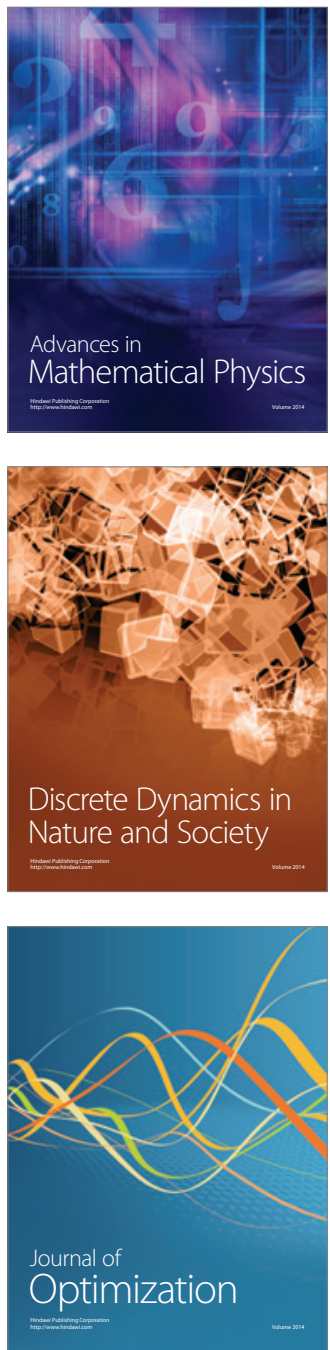